\title{
Dynamics Behaviors of a Laser Produced Plasma: Theoretical Approach
}

\author{
L. R. Manea ${ }^{1}$, C. Nejneru' ${ }^{2}$ D. Mătăsaru ${ }^{3}$, C. I. Axinte $^{2}$, M. Agop ${ }^{4,5}$ \\ ${ }^{1}$ Faculty of Textile, Leather Engineering and Industrial Management, "Gheorghe Asachi" \\ Technical University of Iasi, Iasi, Romania \\ ${ }^{2}$ Faculty of Materials Science and Engineering, "Gheorghe Asachi” Technical University of Iasi, Iași, Romania \\ ${ }^{3}$ Department of Electronics and Telecommunication, "Gheorghe Asachi" Technical \\ University of Iasi, Iași, Romania \\ ${ }^{4}$ Lasers, Atoms and Molecules Physics Laboratory, University of Science and Technology, Lille, France \\ ${ }^{5}$ Physics Department, "Gheorghe Asachi” Technical University of Iasi, Iasi, Romania \\ Email:m.agop@yahoo.com
}

Received April 24, 2013; revised May 26, 2013; accepted June 24, 2013

Copyright (C) 2013 L. R. Manea et al. This is an open access article distributed under the Creative Commons Attribution License, which permits unrestricted use, distribution, and reproduction in any medium, provided the original work is properly cited.

\begin{abstract}
Assuming that plasma particles are moving on continuous and non-differentiable curves, some dynamic properties in plasma ablation are analyzed via scale-relativity theory: the splitting of plasma plume, multi-peak structures, at various distances from the target surface and plasma oscillations through self-similarity. Our theoretical results are in good agreement with the experimental ones.
\end{abstract}

Keywords: Plasma Ablation; Fractal Curves; Plume Splitting; Plasma Oscillations

\section{Introduction}

The interaction of high-power laser radiation with target materials is a topic of current interest in various fields such as material-processing, plasma physics, analytical sciences, etc. [1,2]. Laser ablation implies a series of complex phenomena: interaction of laser radiation with a solid target [3], laser beam absorption in ablation plume [4], hydrodynamics and electrical processes in the generated transient plasma $[5,6]$, etc.

Correspondingly, theoretical models describing the dynamics related to these complex phenomena become sophisticated and ambiguous [1,2]. However, the situation can be standardized taking into account that the elementary processes induced by laser-matter interaction impose various temporal resolution scales [7,8], and that the pattern evolution imposes different degrees of freedom e.g.: from one, at the initial stages, to three at the final stages of the patterns induced by the laser-produced plasma [9]. In the present paper various theoretical aspects of a laser-ablation plasma dynamics (generation of two plasma structures, multi-peak structure for various distances from the target surface, plasma-ablation oscillations through self-similarity, etc.) were analyzed using the SR theory.

\section{Hallmarks of Non-Differentiability}

For developing our theoretical model, we take into account that, in plasma-ablation, deterministic chaos arises in association with spatio-temporal structures emergence. For temporal scales that are large with respect to the inverse of the highest Lyapunov exponent, the deterministic trajectories can be replaced by collections of potential trajectories and the concept of definite positions by that of probability density. This concept was introduced in the framework of the scale relativity (SR) theory [10-12], which states that the particles movement takes place on continuous but non-differentiable curves (fractal curves). Subsequently, all physical phenomena become dependent not only on the spatio-temporal coordinates but also on the spatio-temporal scales. Thus the non-differentiability become a fundamental characteristic of the plasma-ablation dynamics.

Non-differentiability implies the following [10-17]:

1) A continuous and non-differentiable curve (or almost nowhere differentiable) is explicitly scale dependent. Moreover, its length tends to infinity, when the scale interval tends to zero. Consequently, according to Mandelbrot's concept, a continuous and non-differentiable space will be a fractal space [18]; 
2) Physical quantities will be expressed through fractal functions, namely those functions depending on space-time coordinates as well as on resolution scale. The invariance of the physical quantities in relation with the resolution scale generates special types of transformations that are called resolution scale transformations. Let us now explain the above statements through an example. We can choose the fractal normalized currentvoltage characteristic in the form [19]:

$$
\bar{\phi}=\bar{I}\left(1+\frac{\bar{a}}{1+\bar{I}^{2}}\right)
$$

where $\bar{\phi}$ is the fractal normalized voltage, $\bar{I}$ is the fractal normalized current and $\bar{a}$ is a parameter depending on scale resolution. This relation induces conduction bistability (see Figure 1) as follows:

- the restriction $\bar{a} \geq 8$ implies bistability;

- the value of $\bar{a}$ sets the scale resolution through the ionization and recombination rates;

- once $\bar{a}$ is fixed (with $\bar{a} \geq 8$ ), for values of the fractal normalized current in the interval $\mathrm{AB}$ on the characteristic (see Figure 1) the fractal normalized voltage can have two distinct stable values;

- conduction bistability is associated with the negative differential resistance (or hysteresis);

- since $\bar{\phi}$ and $\bar{I}$ are fractal functions (relation (1)) they can exhibit the property of self-similarity. Consequently, conduction bistability in Figure 1 can occur at any scale resolution (i.e. for different ionization and recombination rates). As a result, a correspondence between multiplicity order of the double layer and the one of conduction bistability is likely to occur.

3) Although the local differential time invariance is broken, it can still be recovered through the complex operator [15-17]:

$$
\frac{\hat{\partial}}{\partial t}=\frac{\partial}{\partial t}+\hat{\boldsymbol{V}} \cdot \nabla-\mathrm{i} D(\mathrm{~d} t)^{\left(2 / D_{F}\right)-1} \Delta
$$

where

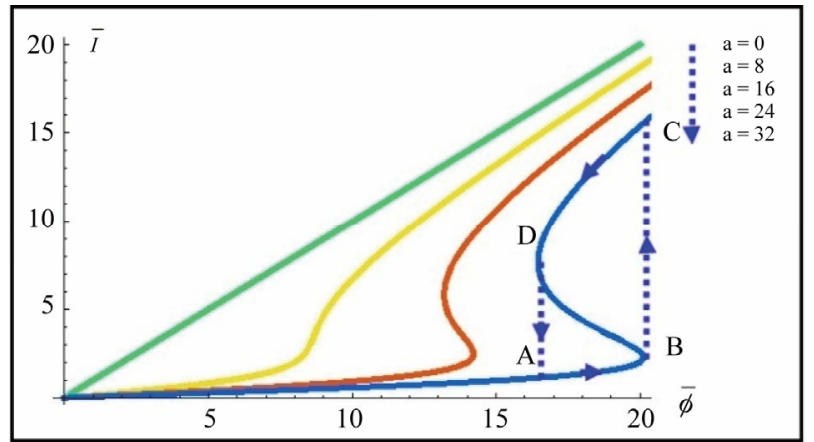

Figure 1. Theoretical dependence of the fractal normalized current on the fractal normalized potential.

$$
\hat{\boldsymbol{V}}=\boldsymbol{V}-\mathrm{i} \boldsymbol{U}
$$

is the complex speed field and $\nabla, \Delta$ are the usual operators. The real part $\boldsymbol{V}$ of the complex speed field $\hat{\boldsymbol{V}}$ represents the standard classical speed which is differentiable and does not depend on resolution, $\mathrm{d} t$, while the imaginary part $\boldsymbol{U}$ is a new quantity arising from fractality, which is non-differentiable and resolution-dependent. Quantity $D$ is Nottale's coefficient and corresponds to the fractal—non-fractal transition while $D_{F}$ is the fractal dimension of motion curves;

4) Plasma-ablation particles may be reduced to and identified with their own trajectories (i.e. their geodesics), so that the complex system should behave as a special "fluid" lacking interaction via geodesics in a non-differentiable (fractal) space. The equation of geodesics (a generalization of Newton's first principle for motion of fractal curves) can be written in the form [15-17]:

$$
\frac{\hat{\partial} \hat{\boldsymbol{V}}}{\partial t}=\frac{\partial \hat{\boldsymbol{V}}}{\partial t}+(\hat{\boldsymbol{V}} \cdot \nabla) \hat{\boldsymbol{V}}-\mathrm{i} D(\mathrm{~d} t)^{\left(2 / D_{F}\right)-1} \Delta \hat{\boldsymbol{V}}=0
$$

This means that the global complex acceleration field $\hat{\partial} \boldsymbol{V} / \partial t$ depends on the local complex acceleration field, $\partial_{t} \hat{\boldsymbol{V}}$, on the non-linear (convective), $(\hat{\boldsymbol{V}} \cdot \nabla) \hat{\boldsymbol{V}}$ and dissipative, $\Delta \hat{\boldsymbol{V}}$ terms. Moreover, the fractal fluid becomes viscoelastic or hysteretic, i.e. the fractal fluid will be endowed with memory. Such a result is in agreement with the opinion given in refs. [1,2]: the fractal fluid can be described through Kelvin-Voight or Maxwell rheological models using complex quantities, e.g. the complex speed field, the complex acceleration field etc. In addition, relation (4) is a Navier-Stokes type equation with an imaginary viscosity coefficient $\eta_{0}=\mathrm{i} D(\mathrm{~d} t)^{\left(2 / D_{F}\right)-1}$.

\section{Fractal Hydrodynamic Model}

If the motions of the fractal fluid are irrotational, i.e. $\nabla \times \hat{\boldsymbol{V}}=0$, we can choose $\hat{\boldsymbol{V}}$ having the form:

$$
\hat{\boldsymbol{V}}=-2 \mathrm{i} D(\mathrm{~d} t)^{\left(2 / D_{F}\right)-1} \nabla \ln \psi
$$

with $\ln \psi$ the scalar potential of the complex speed.

For $\psi=\sqrt{\rho} \mathrm{e}^{\mathrm{i} S}$, with $\sqrt{\rho}$ the amplitude and $S$ the phase of $\psi$, the complex speed field (5) takes the explicit form:

$$
\begin{aligned}
\hat{\boldsymbol{V}}= & 2 D(\mathrm{~d} t)^{\left(2 / D_{F}\right)-1} \nabla S \\
& -\mathrm{i} D(\mathrm{~d} t)^{\left(2 / D_{F}\right)-1} \nabla \ln \rho \\
\boldsymbol{V}= & 2 D(\mathrm{~d} t)^{\left(2 / D_{F}\right)-1} \nabla S \\
\boldsymbol{U}= & D(\mathrm{~d} t)^{\left(2 / D_{F}\right)-1} \nabla \ln \rho
\end{aligned}
$$


Substituting (6a)-(6c) in (4) and separating the real and the imaginary parts up to an arbitrary phase factor which may be zero by a suitable choice of the phase of $\psi$, we shall obtain:

$$
\begin{aligned}
& m_{0}\left(\frac{\partial \boldsymbol{V}}{\partial \boldsymbol{t}}+(\boldsymbol{V} \cdot \nabla) \boldsymbol{V}\right)=-\nabla Q \\
& \frac{\partial \rho}{\partial t}+\nabla \cdot(\rho \boldsymbol{V})=0
\end{aligned}
$$

with $Q$ the fractal potential,

$$
\begin{aligned}
Q & =-2 m_{0} D^{2}(\mathrm{~d} t)^{\left(4 / D_{F}\right)-2} \frac{\Delta \sqrt{\rho}}{\sqrt{\rho}} \\
& =-\frac{m_{0} \boldsymbol{U}^{2}}{2}-m_{0} D(\mathrm{~d} t)^{\left(2 / D_{F}\right)-1} \nabla \cdot \boldsymbol{U}
\end{aligned}
$$

and $m_{0}$ the rest mass of the fractal fluid particle. Equation (7a) is the momentum conservation law, Equation (7b) is the density conservation law. They both define the fractal hydrodynamic model (FHM).

Now, certain conclusions are evident:

1) Any particle is in permanent interaction with the "sub-fractal level" through the fractal potential $Q$;

2) The "sub-fractal level" is identified with a nonrelativistic fractal fluid described by the probability density and the momentum conservation laws - see relations $((7 a),(7 b))$. These equations correspond to the generalised quantum hydrodynamic model (GQHM). Indeed, for motions that can be described via fractal curves in fractal dimension $D_{F}=2$ at Compton scale, $D=\hbar / 2 m_{0}$ with $\hbar$ the reduced Planck constant, the FHM reduces to a quantum hydrodynamic model (QHM). In this last case the "sub-fractal level" is identified with "sub-quantum level" [10-12].

3) The fractal potential (8) results from non-differentiability and should be considered as a kinetic term and not as a potential one. Moreover, the fractal potential (8) can generate a viscosity stress tensor type [15-17].

$$
\begin{aligned}
\hat{\sigma}_{i l} & =m_{0} D^{2}(\mathrm{~d} t)^{\left(4 / D_{F}\right)-2}\left[\nabla_{i} \rho \nabla_{l} \rho-\left(\nabla_{i} \rho \nabla_{l} \rho / \rho\right)\right] \\
& =\eta\left(\frac{\partial U_{i}}{\partial x_{l}}+\frac{\partial U_{l}}{\partial x_{i}}\right), \\
\eta & =\frac{1}{2} m_{0} \rho D(\mathrm{~d} t)^{\left(2 / D_{F}\right)-1}
\end{aligned}
$$

whose divergence is equal to the usual force density associated with $Q$

$$
\nabla_{i} \hat{\sigma}_{i l}=-\rho \nabla_{l} Q
$$

\section{Numerical Simulations}

Let us now rewrite the equations of FHM for the two- dimensional $(x, y)$ case in the form:

$$
\begin{aligned}
& \frac{\partial}{\partial t}\left(\rho V_{x}\right)+\frac{\partial}{\partial x}\left(\rho V_{x}^{2}\right)+\frac{\partial}{\partial y}\left(\rho V_{x} V_{y}\right)=-\frac{\partial \sigma}{\partial x} \\
& \frac{\partial}{\partial t}\left(\rho V_{y}\right)+\frac{\partial}{\partial x}\left(\rho V_{x} V_{y}\right)+\frac{\partial}{\partial y}\left(\rho V_{y}^{2}\right)=-\frac{\partial \sigma}{\partial y} \\
& \frac{\partial \rho}{\partial t}+\frac{\partial}{\partial x}\left(\rho V_{x}\right)+\frac{\partial}{\partial y}\left(\rho V_{y}\right)=0 \\
& \frac{\partial(\rho e)}{\partial t}+\frac{\partial}{\partial x}\left(\rho e V_{x}\right)+\frac{\partial}{\partial y}\left(\rho e V_{y}\right) \\
& =-\rho e\left(\frac{\partial V_{x}}{\partial x}+\frac{\partial V_{y}}{\partial y}\right)
\end{aligned}
$$

where fractal continuity equation of density energy $(\rho e)$ was added [15-17]. With non-dimensional vario ables,

$$
\begin{aligned}
& \omega t=\tau, \\
& k x=\xi, \\
& k y=\eta, \\
& \frac{V_{x} k}{\omega}=V_{\xi}, \\
& \frac{V_{y} k}{\omega}=V_{\eta}, \\
& \frac{\rho}{\rho_{0}}=N
\end{aligned}
$$

in the case of the ideal gas and with variation $\sigma$ being induced by the density and temperature variations $\nabla \sigma=\alpha \nabla(N \cdot T), \alpha=$ const. , Equations (11a)-(11d) becomes:

$$
\begin{aligned}
& \frac{\partial}{\partial \tau}\left(N V_{\xi}\right)+\frac{\partial}{\partial \xi}\left(N V_{\xi}^{2}\right)+\frac{\partial}{\partial \eta}\left(N V_{\xi} V_{\eta}\right)=-\frac{\partial(N \cdot T)}{\partial \xi} \\
& \frac{\partial}{\partial \tau}\left(N V_{\eta}\right)+\frac{\partial}{\partial \xi}\left(N V_{\xi} V_{\eta}\right)+\frac{\partial}{\partial \eta}\left(N V_{\eta}^{2}\right)=-\frac{\partial(N \cdot T)}{\partial \eta} \\
& \frac{\partial N}{\partial \tau}+\frac{\partial}{\partial \xi}\left(N V_{\xi}\right)+\frac{\partial}{\partial \eta}\left(N V_{\eta}\right)=0 \\
& \frac{\partial(N \cdot T)}{\partial \tau}+\frac{\partial}{\partial \xi}\left(N \cdot T \cdot V_{\xi}\right)+\frac{\partial}{\partial \eta}\left(N \cdot T \cdot V_{\eta}\right) \\
& =-N \cdot T\left(\frac{\partial V_{\xi}}{\partial \xi}+\frac{\partial V_{\eta}}{\partial \eta}\right)
\end{aligned}
$$

In Equations (13a)-(13d) the functional scaling relation, $\alpha k^{2} / \omega^{2}=1$ was considered. In a particular case, if we choose in (12a)-(12c) $\rho_{0}$ corresponding to equilibrium plasma density, $\omega$ to ion plasma frequency, and $k$ to the inverse of Debye length, then $\alpha$ will be the square of the ion-acoustic speed and $\sigma$ the kinetic pressure. 
For numerical integration, the initial conditions,

$$
\begin{gathered}
V_{\xi}(0, \xi, \eta)=0, \\
V_{\eta}(0, \xi, \eta)=0, \\
N(0, \xi, \eta)=1 / 4, \\
T(0, \xi, \eta)=1 / 4, \\
0 \leq \xi \times \eta \leq 1 \times 1
\end{gathered}
$$

and the boundary ones,

$$
\begin{aligned}
& V_{\xi}(\tau, 0, \eta)=0, \\
& V_{\xi}(\tau, 1, \eta)=0, \\
& V_{\eta}(\tau, 0, \eta)=0 \text {, } \\
& V_{\eta}(\tau, 1, \eta)=0, \\
& N(\tau, 0, \eta)=1 / 4, \\
& N(\tau, 1, \eta)=1 / 4 \text {, } \\
& T(\tau, 0, \eta)=1 / 4, \\
& T(\tau, 1, \eta)=1 / 4, \\
& V_{\xi}(\tau, \xi, 0)=0 \text {, } \\
& V_{\xi}(\tau, \xi, 1)=0, \\
& V_{\eta}(\tau, \xi, 0)=0 \text {, } \\
& V_{\eta}(\tau, \xi, 1)=0, \\
& N(\tau, \xi, 0) \\
& =N_{0} \exp \left[-\frac{(\tau-1 / 4)^{2}}{(1 / 4)^{2}}\right] \cdot \exp \left[-\frac{(\xi-1 / 2)^{2}}{(1 / 4)^{2}}\right] \text {, } \\
& N(\tau, \xi, 1)=1 / 4, \\
& T(\tau, \xi, 0) \\
& =T_{0} \exp \left[-\frac{(\tau-1 / 4)^{2}}{(1 / 4)^{2}}\right] \cdot \exp \left[-\frac{(\xi-1 / 2)^{2}}{(1 / 4)^{2}}\right], \\
& T(\tau, \xi, 1)=1 / 4 .
\end{aligned}
$$

are considered. In the boundary condition ((15m), (15o)) we assumed that the laser pulse which "hits" the target induces a plasma source that has a spatial-temporal Gaussian profile, similarly with the laser beam. $N_{0}$ is the maximum normalized atom density, while $T_{0}$ is the maximum normalized temperature which is assumed to be proportional with the laser beam energy, by preserving the number of total released atoms.

The equation system (13a)-(13d) with the initial con- ditions (14a)-(14e) and the boundary ones (15a)-(15p) was numerically integrated via finite differences [20]. In Figures 2(a)-(c) space-dependence of the normalized density, $N$, normalized temperature, $T$, and in Figures 3(a)-(c) of the normalized velocities, $V_{\xi}$ and $V_{\eta}$, are given for the initial conditions, $T_{0}=0.4, N_{0}=1$ and various normalized times: Figures 2(a) and 3(a) $-\tau=0.29$, Figures 2(b) and 3(b) $-\tau=0.57$, Figures 2(c) and 3(c) $-\tau$ $=0.88$.

The following results are obtained: 1) the generation of two plasma structures-see Figures 2(b), 3(b); 2) the symmetry of the normalized speed, $V_{\xi}$, according to the axis symmetry of the space-time Gaussian; 3) shock waves and vertices at the plume periphery for the normalized speed field, $V_{\eta}$.

These data are in agreement with the experimental images at various evolution stages [5,6,21]. Moreover, if the current density of the particle is plotted on the symmetry axis $(\xi=1 / 2)$ —see Figures 4(a)-(c), for various distances from the target surface, a multi-peak structure as in $[5,6]$ can be noticed. Increasing the value of the control parameter, $\eta$, we conclude: 1 ) the arrival time of the first peak decreases; 2) the ratio between the first and the second maximum increases. This is in agreement with the experimental data according to which the particles are gradually transferred from the fast into the slow part [22-24]; 3) the magnitude of the first maximum decreases as a consequence of lateral expansion. Therefore we conclude that plume splitting is a hydrodynamic process similar to Gaussian disturbance emission.

\section{Self-Similarity in Plasma Ablation}

Neglecting the convection $\hat{\boldsymbol{V}} \cdot \nabla \hat{\boldsymbol{V}}$, Equation (4) takes the form:

$$
\frac{\partial \hat{\boldsymbol{V}}}{\partial t}-\mathrm{i} D(\mathrm{~d} t)^{\left(2 / D_{F}\right)-1} \Delta \hat{\boldsymbol{V}}=\mathbf{0}
$$

or, separating the resolution scales,

$$
\frac{\partial \boldsymbol{V}}{\partial t}-D(\mathrm{~d} t)^{\left(2 / D_{F}\right)-1} \Delta \boldsymbol{U}=0
$$

for the differentiable scale, and

$$
\frac{\partial \boldsymbol{U}}{\partial t}-D(\mathrm{~d} t)^{\left(2 / D_{F}\right)-1} \Delta \boldsymbol{V}=0
$$

for the fractal scale. Velocity fields are totally separated, firstly applying Equations (17) and (18) to the $\Delta$ operator, i.e.

$$
\frac{\partial}{\partial t}(\Delta \boldsymbol{V})-D(\mathrm{~d} t)^{\left(2 / D_{F}\right)-1} \Delta^{2} \boldsymbol{U}=0
$$

and

$$
\frac{\partial}{\partial t}(\Delta \boldsymbol{U})+D(\mathrm{~d} t)^{\left(2 / D_{F}\right)-1} \Delta^{2} \boldsymbol{V}=0
$$

then, by substituting the dissipative terms via Equations 


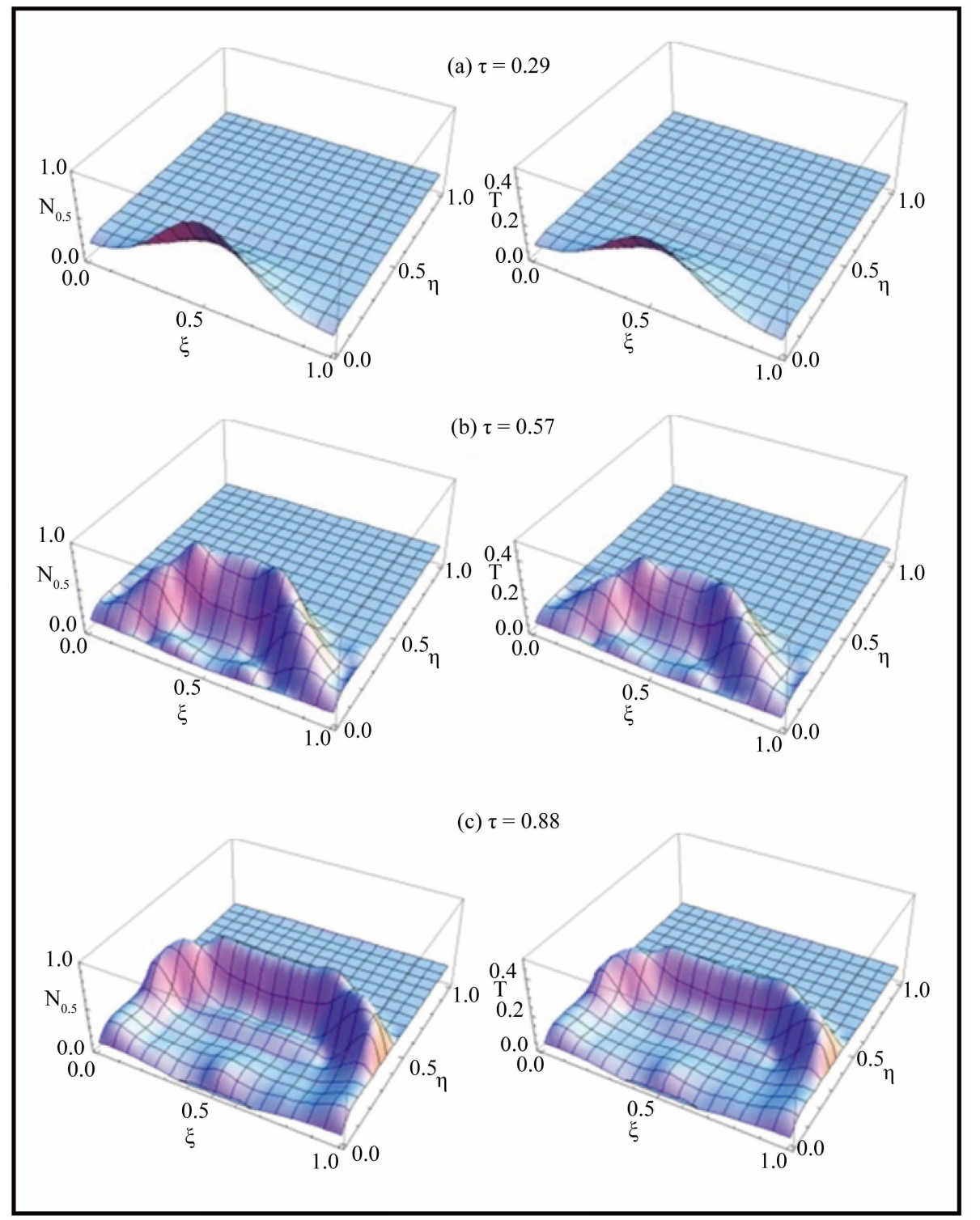

Figure 2. (a)-(c) Space-dependence of the normalized density, $N$, and normalized temperature, $T$, resulting from numerical simulations in Equations (13a)-(13d) for normalized initial conditions, $T_{0}=0.4, N_{0}=1$ and various normalized times: (a) $\tau=$ 0.29 ; (b) $\tau=0.57$; (c) $\tau=0.88$.

(17) and (18), the Kirchhoff-type equations [25] result:

$$
\left(\frac{\partial^{2}}{\partial t^{2}}+D(\mathrm{~d} t)^{\left(4 / D_{F}\right)-2} \Delta^{2}\right)\left(\begin{array}{l}
\boldsymbol{V} \\
\boldsymbol{U}
\end{array}\right)=\left(\begin{array}{l}
0 \\
0
\end{array}\right)
$$

For the one-dimensional case, the previous equations with the substitutions:

$$
\begin{aligned}
\frac{x}{L} & =\bar{\xi}, \\
\frac{t}{T} & =\bar{\tau}, \\
\frac{L^{4}}{T^{2}} & =D^{2}(\mathrm{~d} t)^{\left(4 / D_{F}\right)-2},
\end{aligned}
$$

$$
(V ; U) \equiv \overline{\mathrm{K}}_{\mathrm{i}}(\bar{\xi}, \bar{\tau}), i=1,2
$$

take the unitary form [26]:

$$
L^{4} \frac{\partial^{4} \overline{\mathbf{K}}_{\mathrm{i}}}{\partial \bar{\xi}^{4}}+T^{2} \frac{\partial^{2} \overline{\mathbf{K}}_{\mathrm{i}}}{\partial \bar{\tau}^{2}}=0
$$

We impose "clamping" conditions at $\bar{\xi}=1$ for Equation (23):

$$
\begin{gathered}
\frac{\partial^{2} \overline{\mathrm{K}}_{\mathrm{i}}(1, \bar{\tau})}{\partial \bar{\xi}^{2}}=0, \\
\frac{\partial^{3} \overline{\mathrm{K}}_{\mathrm{i}}(1, \bar{\tau})}{\partial \bar{\xi}^{3}}=0
\end{gathered}
$$




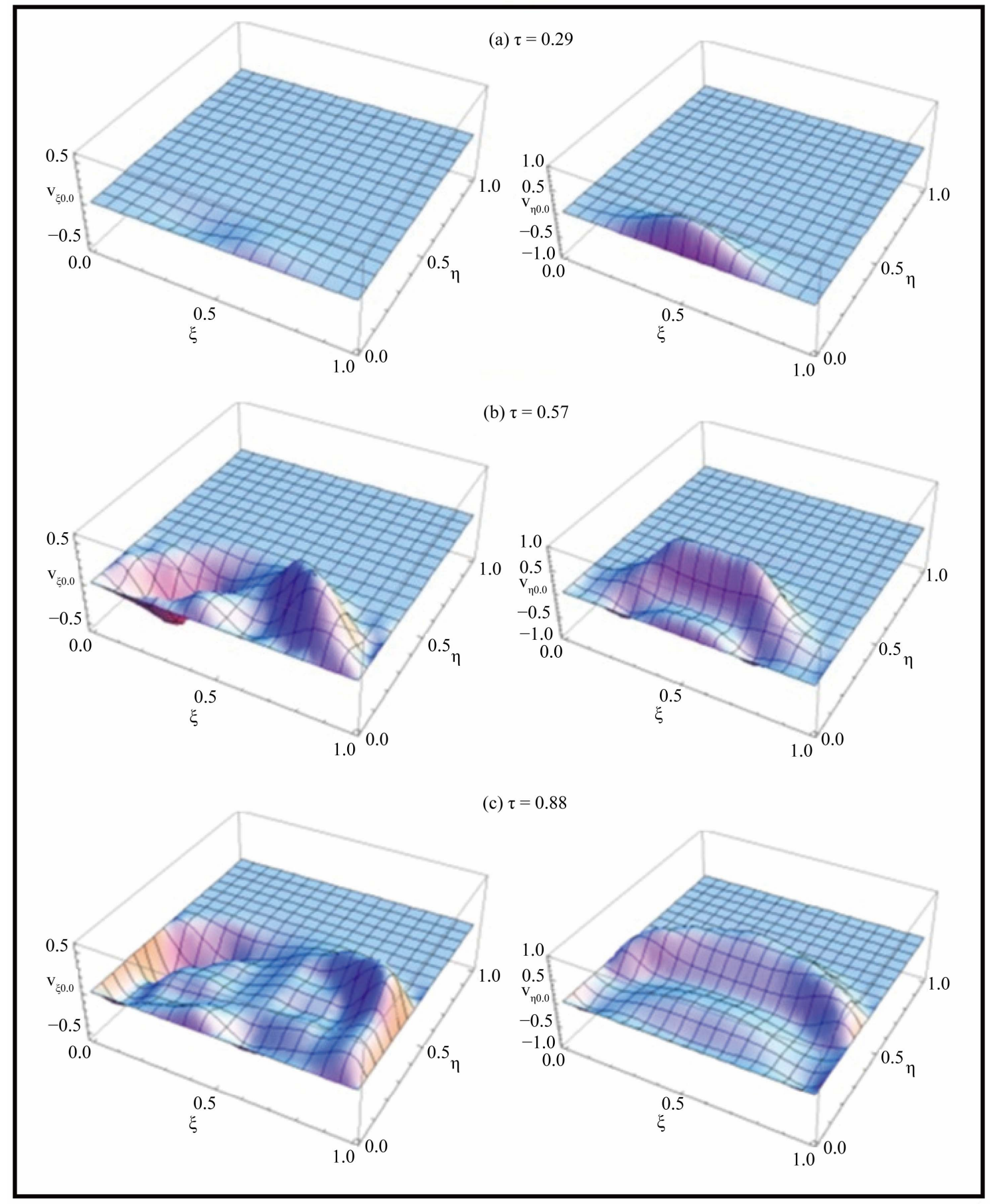

Figure 3. (a)-(c) Space-dependence of normalized velocities, $V_{\xi}$ and $V_{\eta}$, resulting from numerical simulations of Equations (13a)-(13d) for normalized initial conditions, $T_{0}=0.4, N_{0}=1$ and various normalized times: (a) $\tau=0.29 ;$ (b) $\tau=0.57$; (c) $\tau$ $=0.88$.

and for boundary conditions at $\bar{\xi}=0$ :

$$
\overline{\mathrm{K}}_{\mathrm{i}}(0, \bar{\tau})=0, \frac{\partial \overline{\mathrm{K}}_{\mathrm{i}}(0, \bar{\tau})}{\partial \bar{\xi}}=0
$$

These four boundary conditions in $\bar{\xi}$ associated with the two initial ones

$$
\begin{aligned}
& \overline{\mathrm{K}}_{\mathrm{i}}(\bar{\xi}, 0)=\overline{\mathrm{K}}_{\mathrm{i} 0}, \\
& \frac{\partial \overline{\mathrm{K}}_{\mathrm{i}}(\bar{\xi}, 0)}{\partial \bar{\tau}}=0
\end{aligned}
$$

implies a unique solution $\overline{\mathrm{K}}_{\mathrm{i}}(\bar{\xi}, \bar{\tau})$ to Equation (23) 


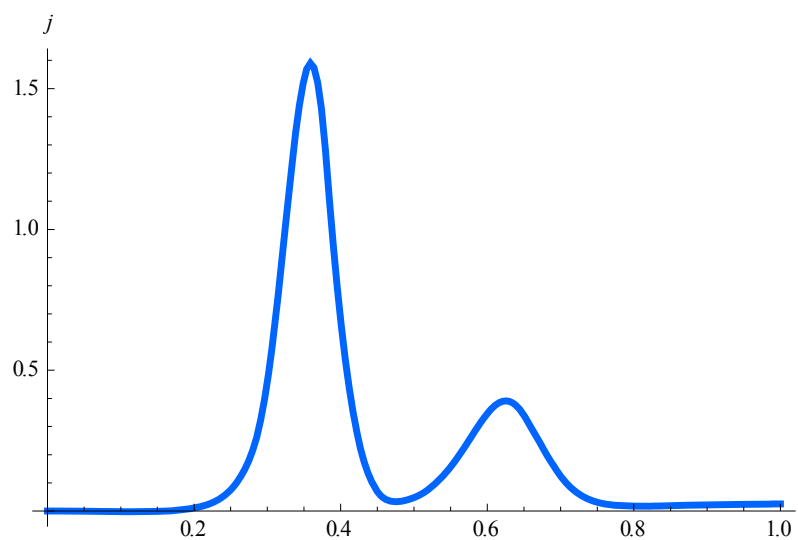

(a)

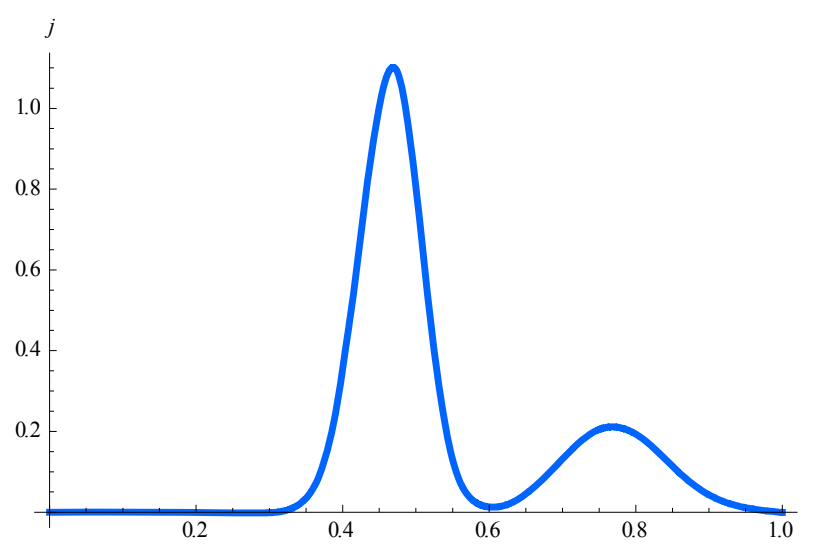

(b)

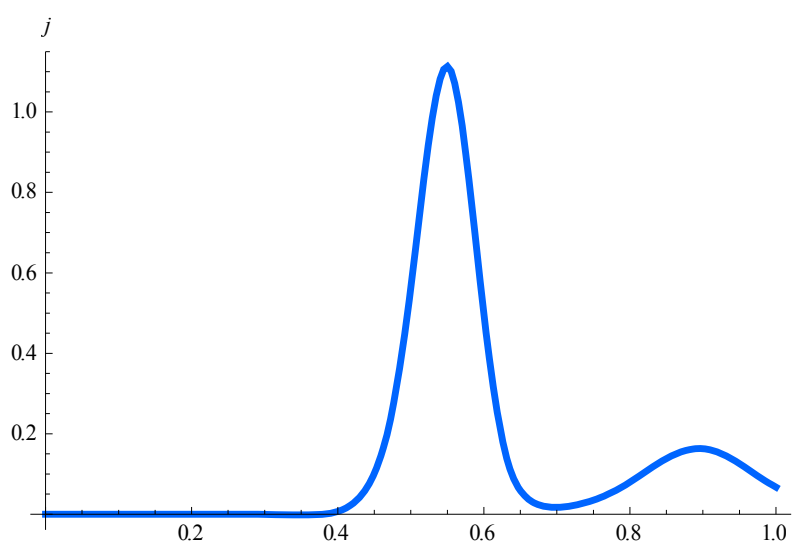

(c)

Figure 4. (a)-(c) Time-dependence of the particle current density on the symmetry axis $(\xi=1 / 2)$ resulting from numerical simulations for various normalized distances from the target surface: (a) $\eta=0.19$; (b) $\eta=0.28$; (c) $\eta=0.42$.

\section{- see Figure 5.}

Owing to scaling $x \sim L \sqrt{t / T}$, we find a solution for Equation (23) in the form:

$$
\overline{\mathrm{K}}_{\mathrm{i}}(\bar{\xi}, \bar{\tau})=\overline{\mathrm{K}}_{\mathrm{i} 0} u_{i}(\bar{\eta})
$$

where the self-similarity variable is:

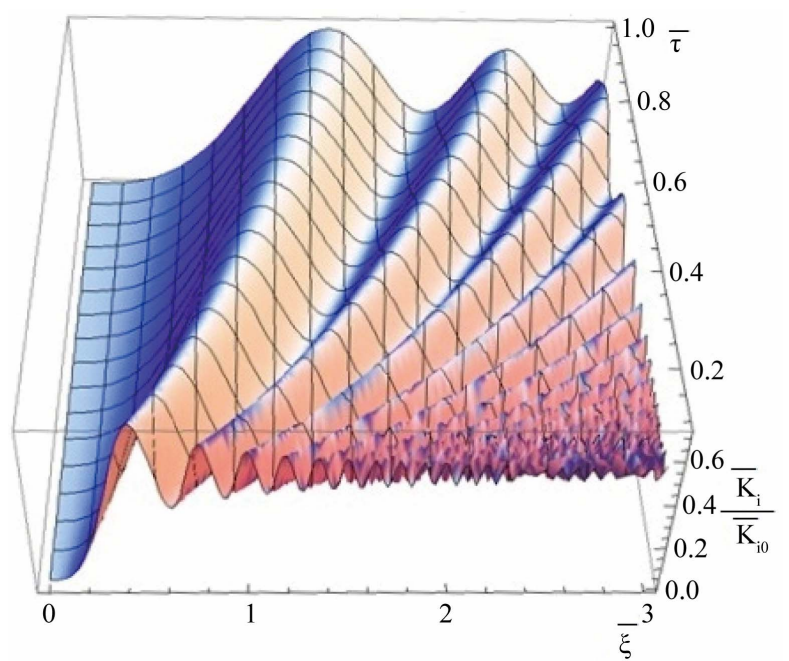

Figure 5. Numerical solution of the Kirchhoff Equation (23) with "clamped-free" unitary conditions, for a uniform initial $\overline{\mathbf{K}}_{\mathrm{i} 0} \cdot \overline{\mathbf{K}}_{\mathrm{i}}(\mathbf{0}, \tau)$ relaxes to zero within the first few timesteps.

$$
\bar{\eta}=(\bar{\xi} / \sqrt{\bar{\tau}})=(x / L) / \sqrt{t / T}=x / \sqrt{\gamma t}
$$

The boundary condition for $u(\bar{\eta})$ derives from $\overline{\mathrm{K}}_{\mathrm{i}}$ conditions:

$$
\begin{gathered}
u_{i}(0)=0, \\
u_{i}^{\prime}(0)=0, \\
u_{i}(+\infty) \rightarrow 1
\end{gathered}
$$

Substituting this self-similar form of $\overline{\mathrm{K}}_{\mathrm{i}}(\bar{\xi}, \bar{\tau})$ in Equation (23), the following equation for the self-similar solution $u_{i}(\bar{\eta})$ results:

$$
4 \frac{\mathrm{d}^{4} u_{i}(\bar{\eta})}{\mathrm{d} \bar{\eta}^{4}}+\bar{\eta}^{2} \frac{\mathrm{d}^{2} u_{i}(\bar{\eta})}{\mathrm{d} \bar{\eta}^{2}}+3 \bar{\eta} \frac{\mathrm{d} u_{i}(\bar{\eta})}{\mathrm{d} \bar{\eta}}=0
$$

The additional condition:

$$
\frac{\mathrm{d}^{2} u_{i}(0)}{\mathrm{d} \bar{\eta}^{2}}=0
$$

combined with the previous ones, gives a unique selfsimilar solution to Equation (30):

$$
2 \overline{\mathrm{K}}_{\mathrm{i}}(\bar{\xi}, \bar{\tau})=2 \overline{\mathrm{K}}_{\mathrm{i} 0}\left(\frac{\bar{\eta}}{\sqrt{2 \pi}}\right)
$$

see Figure 6, where the Fresnel sine integral,

$$
S(x)=\int_{0}^{x} \sin \left(\pi y^{2} / 2\right) d y
$$

from the theory of diffraction has been introduced.

Relation (32) describes an oscillation via a self-similar solution $x \sim \sqrt{\gamma t}$. This reflects the dispersive nature of Equation (23). 


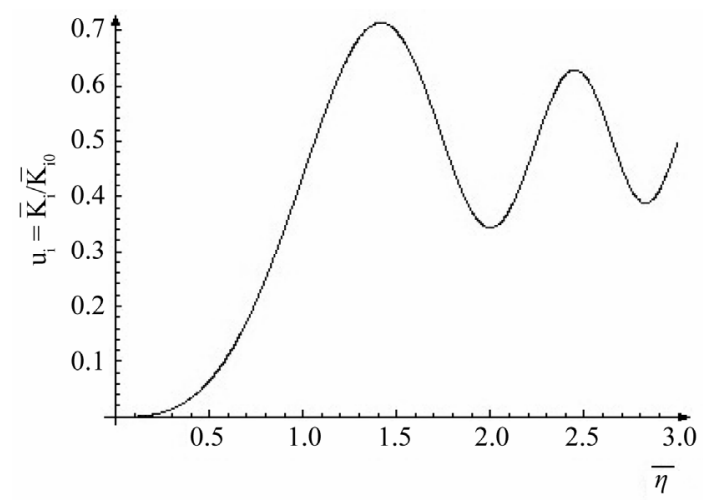

Figure 6. Self-similar solution (32) as a function of $\bar{\eta}$.

Accordingly, self-similarity generates oscillations in plasma-ablation. This result is experimentally confirmed in $[5,6,21,22]$.

\section{Conclusions}

The main conclusions of the present paper are the following: 1) the plasma expansion was theoretically analyzed assuming that the particle moves on continuous and non-differentiable curves; 2) a fractal hydrodynamic model containing the density and momentum conservation equations was built. Fractality is introduced via fractal potential; 3) suposing that the fractal potential is connected with the ideal gas pressure, the ablation plasma expansion is studied through numerical simulations. The splitting of plasma plume, multi-peak structures at various distances from the target surface can be noticed; 4) in the absence of convection, oscillations via self-similarity are induced in plasma-ablation.

Theoretical data are in good agreement with the experimental ones.

\section{Acknowledgements}

This paper was realised with the support of Posdru Cuantumdoc "Doctoral Studies for European Performances in Research and Inovation". ID79407 project funded by the European Social Found and Romanian Government.

\section{REFERENCES}

[1] C. R. Phipps, Ed., "Laser Ablation and Its Applications," Springer, New York, 2006.

[2] L. J. Radziemski and D. A. Cramers, "Laser Induced Plasma and Applications," Dekker, New York, 1989.

[3] A. Peterlongo, A. Miotello and R. Kelly, Physical Review E, Vol. 50, 1994, pp. 4716-4727. doi:10.1103/PhysRevE.50.4716

[4] R. Jordan and J. G. Lunney, Applied Surface Science, Vol. 215,1998 , pp. $127-129$. doi:10.1016/S0169-4332(97)00775-7
[5] S. Gurlui, M. Agop, P. Nica, M. Ziskind and C. Focşa, Physical Review E, Vol. 78, 2008, Article ID: 026405. doi:10.1103/PhysRevE.78.026405

[6] C. Ursu, O. G. Pompilian, S. Gurlui, P. Nica, M. Agop, M. Dudeck and C. Focşa, Applied Physics A, Vol. 15, No. 28, 2010 .

[7] M. Murakami, Y. G. Kang, K. Nishihara and H. Nishimura, Physics of Plasmas, Vol. 12, 2005, Article ID: 062706. doi:10.1063/1.1928247

[8] P. Mora, Physical Review Letters, Vol. 90, 2003, Article ID: 185002. doi:10.1103/PhysRevLett.90.185002

[9] N. M. Bulgakova, A. V. Bulgakov and O. F. Bobrenok, Physical Review E, Vol. 62, 2000, pp. 5624-5635. doi:10.1103/PhysRevE.62.5624

[10] L. Nottale, "Fractal Space-Time and Microphysics: Towards a Theory of Scale Relativity," World Scientific, Singapore, 1993. doi:10.1142/1579

[11] L. Nottale, "Scale Relativity and Fractal Space-Time-A New Approach to Unifying Relativity and Quantum Mechanics," Imperial College Press, London, 2011.

[12] L. Nottale, International Journal of Modern Physics A, Vol. 4, 1989, pp. 5047-5117. doi:10.1142/S0217751X89002156

[13] M. S. El Naschie, O. E. Rossler and I. Prigogine, "Quantum Mechanics, Diffusion and Chaotic Fractals," Elsevier, Oxford, 1995.

[14] P. Weibel, G. Ord and O. E. Rosler, "Space Time Physics and Fractility," Springer, New York, 2005. doi:10.1007/3-211-37848-0

[15] M. Agop, N. Forna, I. Casian-Botez and C. Bejenariu, Journal of Computational and Theoretical Nanoscience, Vol. 5, 2008, pp. 483-489.

[16] I. Casian-Botez, M. Agop, P. Nica, V. Paun and G. V. Munceleanu, Journal of Computational and Theoretical Nanoscience, Vol. 7, 2010, pp. 2271-2280. doi:10.1166/jetn.2010.1608

[17] G. V. Munceleanu, V. P. Paun, I. Casian-Botez and M. Agop, International Journal of Bifurcation and Chaos, Vol. 21, 2011, pp. 603-618. doi:10.1142/S021812741102888X

[18] B. Mandelbrot, "The Fractal Geometry of Nature," W. H. Freeman, New York, 1983.

[19] M. Agop, P. Nica, O. Niculescu and D.-G. Dumitru, Journal of the Physical Society of Japan, Vol. 81, 2012, Article ID: 064502. doi:10.1143/JPSJ.81.064502

[20] O. C. Zinkiewikz, R. L. Taylor and J. Z. Zhu, "The Finite Element Method: Its Basis and Fundamentals," 6th Edition, Elsevier, Butterworth-Heinemann, 2005.

[21] P. Nica, M. Agop, S. Gurlui, C. Bejinariu and C. Focsa, Japanese Journal of Applied Physics, Vol. 51, 2012, Article ID: 106102. doi:10.1143/JJAP.51.106102

[22] P. Nica, M. Agop, S. Gurlui and C. Focsa, Europhysics Letters, Vol. 89, 2010, Article ID: 65001. doi:10.1209/0295-5075/89/65001

[23] P. Nica, M. Agop, S. Miyamoto, S. Amano, A. Nagano, T. Inoue, E. Poll and T. Mochizuki, European Physical Jour- 
nal D, Vol. 60, 2010, pp. 317-323. doi:10.1140/epjd/e2010-00217-2

[24] P. Nica, S. Miyamoto, S. Amano, T. Inoue, A. Shimoura and T. Mochizuki, Physics Letters A, Vol. 370, 2007, pp. 154-157. doi:10.1016/j.physleta.2007.05.105

[25] B. D. Coleman, E. H. Dill, M. Lembo, Z. Lu and I. To- bias, Archive for Rational Mechanics and Analysis, Vol. 121, 1993, pp. 339-359. doi:10.1007/BF00375625

[26] B. Audoly and S. Neukirch, Physical Review Letters, Vol. 95, 2005, Article ID: 095505. doi:10.1103/PhysRevLett.95.095505 